\title{
"Presemantic" Cognition in Semantic Dementia: Six Deficits in Search of an Explanation
}

\author{
Karalyn Patterson ${ }^{1}$, Matthew A. Lambon Ralph ${ }^{2}$, Elizabeth Jefferies ${ }^{2}$, \\ Anna Woollams ${ }^{1}$, Roy Jones ${ }^{3}$, John R. Hodges ${ }^{1}$, and Timothy T. Rogers ${ }^{1,4}$
}

\begin{abstract}
"Oh, sir, you must be well aware that life is full of endless absurdities which do not even have to appear plausible, since they are true."
\end{abstract}

—From Six Characters in Search of an Author, by Luigi Pirandello (1921)

\begin{abstract}
On the basis of a theory about the role of semantic knowledge in the recognition and production of familiar words and objects, we predicted that patients with semantic dementia would reveal a specific pattern of impairment on six different tasks typically considered "pre-" or "non-" semantic: reading aloud, writing to dictation, inflecting verbs, lexical decision, object decision, and delayed copy drawing. The prediction was that all tasks would reveal a frequency-by-typicality interaction, with patients performing especially poorly on lower-frequency items with atypical structure (e.g., words with an atypical spelling-to-sound relationship; objects with an atypical feature for their class, such as the hump on a camel, etc). Of 84 critical
\end{abstract}

\section{INTRODUCTION}

Semantic dementia (SD) is a cognitive syndrome associated with progressive, relatively focal, bilateral (although often asymmetrical) degeneration of the anterior temporal lobes, and is characterized by gradual deterioration of conceptual knowledge. SD is commonly described in the literature as a selective disruption to semantic memory, and much of the theoretical attention devoted to this syndrome derives from its apparent selectivity. On the other hand, where this has been evaluated, patients with SD are typically impaired on some cognitive processes that are usually thought to be performed largely without reference to or need for conceptual knowledge: the "presemantic" cognition in the title of this article. Six examples of such impairments in SD are: reading aloud single written words (Fushimi et al., 2003; Funnell, 1996; Patterson \& Hodges, 1992);

\footnotetext{
${ }^{1}$ MRC Cognition \& Brain Sciences Unit, Cambridge, UK, ${ }^{2}$ University of Manchester, UK, ${ }^{3}$ St Martin's Hospital, Bath, UK, ${ }^{4}$ University of Wisconsin, Madison
}

observations (14 patients performing 6 tasks), this prediction was correct in 84/84 cases; and a single component in a factor analysis accounted for $87 \%$ of the variance across seven measures: each patient's degree of impairment on atypical items in the six experimental tasks and a separate composite score reflecting his or her degree of semantic impairment. Errors also consistently conformed to the predicted pattern for both expressive and receptive tasks, with responses reflecting residual knowledge about the typical surface structure of each domain. We argue that these results cannot be explained as associated but unrelated deficits but instead are a principled consequence of a primary semantic impairment.

spelling single spoken words (Graham, Patterson, \& Hodges, 2000; Parkin, 1993); transforming the stem (present tense) forms of verbs into their corresponding past-tense forms (Patterson, Lambon Ralph, Hodges, \& McClelland, 2001; Cortese, Balota, Sergent-Marshall, Buckner, \& Gold, in press); judging whether orthographically well-formed letter strings represent real words, that is, lexical decision (Rogers, Lambon Ralph, Hodges, \& Patterson, 2004; Moss, Tyler, Hodges, \& Patterson, 1995; Diesfeldt, 1992); judging whether line drawings of possible objects represent objects that really exist, that is, object decision (Hovius, Kellenbach, Graham, Hodges, \& Patterson, 2003; Rogers, Lambon Ralph, Hodges, \& Patterson, 2003; Breedin, Saffran, \& Coslett, 1994); and reproducing simple line drawings of familiar objects after a short (e.g., $10 \mathrm{sec}$ ) delay (Bozeat et al., 2003; Lambon Ralph \& Howard, 2000).

The SD deficit in each of these processes follows a specific and common pattern in which-provided that the stimulus materials were selected to enable evaluation of these factors-performance is characterized by a 
strong frequency-by-typicality interaction. That is, if the stimulus item is familiar and/or it has a structural form fairly typical of its domain, SD patients often perform well, demonstrating that the tasks themselves are not problematic. When the patients are asked to recognize or produce lower-familiarity words or pictures with atypical form or structure, on the other hand, accuracy tends to be significantly below normal. Furthermore, in the four of these six tasks that require production (reading aloud, spelling to dictation, past-tense verb inflection, delayed copy of object drawings), the errors also follow a specific and common pattern: Relative to the intended targets, the patients' responses are more typical or "regularized" (Rogers, Lambon Ralph, Garrard, et al., 2004).

The question addressed in this article is how best to account for these additional features of the cognitive profile of SD. One theoretically uninspiring but nevertheless critically important hypothesis is that these are spurious associations arising from the simple fact that brain lesions can produce multiple, unrelated deficits. For example, when an SD patient is invited to read aloud a written word with an atypical spelling-sound correspondence (like sew in English), and mispronounces it in a more typical fashion (sew $\rightarrow$ "sue," rhyming with new: e.g., Funnell, 1996; Patterson \& Hodges, 1992), this might indicate dysfunction in a brain region needed to compute the pronunciation of words with atypical spelling-sound correspondences that is independent of the neurological abnormality causing the patient's semantic deficit. Likewise, when an SD patient performs two-alternative forced-choice (2AFC) object decision and selects the picture of a camel without a hump as the real thing in preference to the humped camel (Rogers, Lambon Ralph, Hodges, \& Patterson, 2003, 2004), such errors could reflect abnormality in the brain region representing presemantic structural descriptions of familiar objects, again separate from the atrophy causing the deterioration of conceptual knowledge. The six processes in focus here are rather disparate in the sense that they include four verbal and two nonverbal tasks, and also comprise two that require only recognition of words or objects and four that involve output in the form of word production or drawing. As a consequence, it is unlikely that all six of these abilities would be disrupted by impairment to a single additional nonsemantic cognitive function. The main alternative interpretations, therefore, seem to be either (i) that all of the deficits can be interpreted as a principled consequence of the primary semantic degradation, or (ii) that these six associated deficits are explained by disruptions to a number of different additional cognitive subsystems. We shall refer to these two positions as It's All Semantic (IAS) and Associated But Unrelated Deficits (ABUD). The ABUD position is perhaps most extensively argued by Coltheart (2004) but is also explicit or inherent in the accounts of many other researchers studying these processes (e.g., Miozzo \& Gordon, 2005; Tyler et al., 2004; Riddoch \& Humphreys, 1987).

This article presents a case for IAS, and we begin with a brief explanation of the basis for predicting that a degraded semantic system should reliably engender these associated deficits (for further detail, see Rogers, Lambon Ralph, Garrard, et al, 2004; Rogers, Lambon Ralph, Hodges, \& Patterson, 2004). The account proposes that, in addition to processes occurring in many other parts of the brain, each of the tasks in focus here recruits a semantic network in the anterior temporal lobes whose participation is necessary for normal performance. The semantic representations proposed do not represent explicit conceptual content (e.g., that canaries are yellow or that knives cut); they are, instead, abstract representations that encode higher-order relations among features and concepts. These representations are structured to enable generalization among similar concepts, which is critical for learning and for responding to new tokens of familiar types. Of equal importance, the semantic representations permit differentiation between similar concepts, which is essential for other sorts of processes such as object naming or knowing that one kind of mushroom is delicious and safe to eat but another similar-looking one is poisonous.

Whereas the semantic system encodes representations that capture conceptual similarity structure (Rogers \& McClelland, 2004), the surface representations that encode input to or output from semantics capture structure that is tailored to particular expressive or receptive domains. For inputs that are generally consistent with the surface structure in some modality-for instance, "standard" four-legged, two-eyed, two-eared, one-tailed, humpless animals, or verbs that form the past tense by adding ed-such structure facilitates processing: The correct surface representations can be computed predominantly through local interactions without much support from semantics. Items that have more idiosyncratic component features (e.g., written words with infrequent letter combinations), or unusual relationships to their representations in other domains (e.g., words with atypical pronunciations for their spellings) are not so well supported by within-modality similarity structure, nor by interaction with other surface forms. Interaction with the semantic system is therefore required for correct processing of these items, particularly if they are not overlearned by virtue of high familiarity. As a result, atypical and less familiar items, in any of these tasks/domains, will suffer when the semantic system is degraded; and their erroneous processing will take a predictable form, with the items being treated as if they embodied the typical characteristics of the domain. These principles have been demonstrated in computational models of word-reading (Plaut, McClelland, Seidenberg, \& Patterson, 1996), past-tense formation (Joanisse \& Seidenberg, 1999), and delayed copy drawing (Rogers, Lambon Ralph, Garrard, et al., 2004). 
The empirical component of this report consists of data from 14 patients with SD, all of whom were tested on all six of the "nonsemantic" tasks. As mentioned above, there is already evidence in the literature for impairment in each of these six abilities in patients with $\mathrm{SD}$, but the IAS account requires more data for the following principal reason: The demonstrations of each of these impairments have largely applied to independent groups of SD patients. A proponent of ABUD might argue that the three SD patients tested on delayed copy drawing in Bozeat et al. (2003), and all shown to be impaired, happened to have additional damage to brain regions crucial for visual structural descriptions of objects, but that other SD patients would not be so characterized. Likewise, the six SD patients in Patterson and Hodges (1992) with deficits in reading words with irregular spelling-sound correspondences might coincidentally have had structural or functional lesions to the lexical route for reading. As there was no overlap between the patients participating in these two studies, the current literature does not reveal whether the three cases in Bozeat et al. had reading deficits like the patients in Patterson and Hodges, nor whether the six cases in the reading study would have had trouble in delayed copying of objects with atypical features.

The principal findings of the study will be reported in three sections dealing with: (1) the patients' accuracy on the four conditions of each of the six tasks relative to control data for the same test; (2) the patients' error types in the four production tasks (the two receptive tasks were both 2AFC, meaning that the only errors that a subject could make were the choices selected by the experimenters); (3) the relationship of each patient's performance on the six tasks to his or her degree of semantic decline. The strongest statements of our predictions with respect to these three aspects of the results are as follows. (1) The patients should be impaired on every one of the tasks, with the pattern of impairment characterized by a large typicality effect and further by a Frequency $\times$ Typicality interaction. (2) The great majority of the patients' errors in productive tasks should be composed of elements that are legitimate for those components of the stimulus in other contexts, and thus, reflect the patients' residual knowledge of typical structure for the relevant domain. (3) Individual levels of accuracy on the atypical stimuli in all tasks should be modulated by the patient's degree of semantic deterioration as indexed by a composite semantic score.

\section{RESULTS}

\section{Accuracy}

Figure 1 displays the proportion of correct responses in the six experimental tasks. Each panel in the figure corresponds to one task and indicates (a) the range of control performance (shown as pale gray rectangles) for each of the four conditions created by crossing frequency or familiarity with regularity or typicality (operationally defined for each task in the Methods section); and (b) the mean and 90\% confidence intervals of the SD patients' performance, separately for the half of the patient group with milder semantic impairment and the half with more severe semantic degradation. For the two receptive tasks, lexical decision and object decision, which were both presented in a $2 \mathrm{AFC}$ paradigm, chance-level performance (0.5) is indicated by a dashed line. For the drawing task, control performance is marked as $100 \%$ in all conditions, because only the features of the drawings produced by every control subject were used as the baseline against which to evaluate the patients' performance.

Control subjects performed all of these tasks with relative ease and with only a modest impact of frequency and typicality on their accuracy. We have not superimposed control means on the control performance range in Figure 1 so as to avoid clutter; but in every task, control performance averaged $\geq 95 \%$ correct, even in the most difficult condition with low-frequency irregular targets. For the two receptive tasks (lexical and object decision), control means and variances were equivalent for regular and irregular items in both frequency conditions.

The patients did not have marked difficulty with any of these tasks per se: In the higher-frequency regular condition on all tasks, even the more severely impaired subgroup scored comfortably above chance in the two forced-choice receptive tasks and mostly above 75\% correct in the four production tasks. The patients' performance, however, was consistently and markedly affected by condition. For the six low-frequency irregular conditions, the 90\% confidence intervals for both the milder and the more semantically impaired subgroup were substantially below the control range. Note that in this condition of the two 2AFC tasks, the confidence intervals for the most semantically impaired cases include chance level for object decision and are entirely below chance for lexical decision. In other words, as demonstrated previously by Rogers, Lambon Ralph, Hodges, and Patterson (2004) and by Bub, Cancelliere, and Kertesz (1985), patients with profoundly degraded conceptual knowledge often select nonwords in preference to lower-frequency real words in lexical decision if the nonword in the pair has a more typical orthographic structure than the word (example: khaki/kackey).

The results of a series of six ANOVAs, each with two within-subject factors (frequency and typicality) and each of these with two levels (high vs. low frequency and typical vs. atypical), are displayed in Table 1. All six tasks yielded a significant main effect of typicality, and five of the six produced significant main effects of frequency plus reliable interactions between these two variables. The one exception in the pattern was delayed copy drawing, which showed a strong typicality effect 
Figure 1. Means and $90 \%$ confidence intervals plotted separately for milder $(n=7)$ and more severely impaired $(n=7)$ patients in the four conditions of each task (HF Reg: high-frequency regular, LF Reg: low-frequency regular, HF Irr: high-frequency irregular, and LF Irr:

low-frequency irregular). The gray rectangles indicate the range of performance for age-matched controls. The dotted lines for lexical and object decision indicate chance-level performance. For all tasks except delayed copy drawing, scores indicate proportion correct in each task condition. For delayed copy drawing, the data indicate the features of the objects in each condition that were produced by the patients as a proportion of those produced by all control participants.

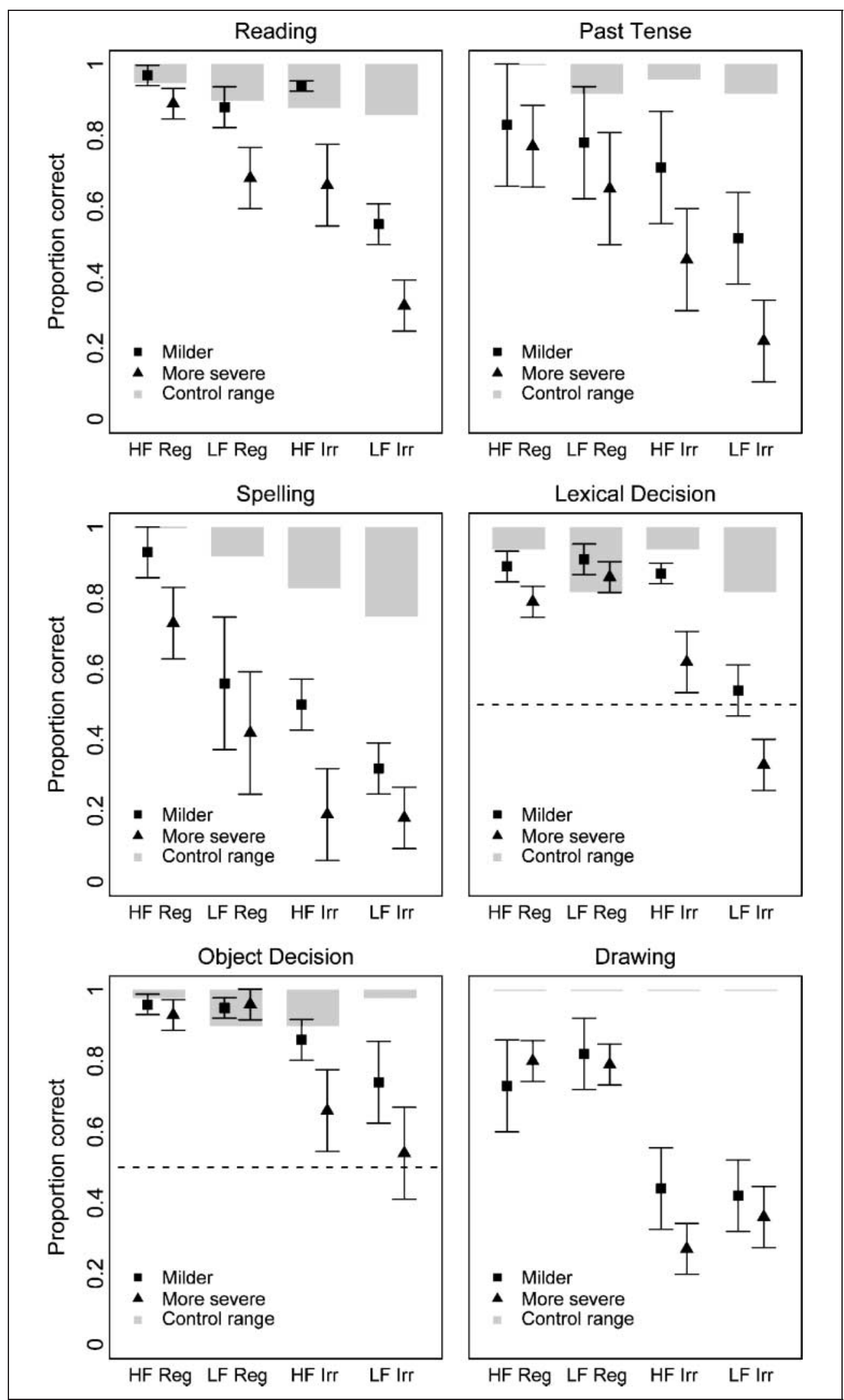


Table 1. Results of Six $2 \times 2$ ANOVAs, Each with $d f=1,12$, Designed to Assess the Significance of the Frequency and Typicality Effects in Each Experimental Task

\begin{tabular}{|c|c|c|c|c|c|c|}
\hline & \multicolumn{2}{|c|}{ Frequency } & \multicolumn{2}{|c|}{ Typicality } & \multicolumn{2}{|c|}{ Frequency $\times$ Typicality } \\
\hline & $F$ ratio & p value & $F$ ratio & p value & $F$ ratio & pvalue \\
\hline Reading & 56.03 & $<.001$ & 82.77 & $<.001$ & 41.25 & $<.001$ \\
\hline Verbs & 28.52 & $<.001$ & 25.64 & $<.001$ & 5.19 & .04 \\
\hline Spelling & 16.21 & .002 & 76.70 & $<.001$ & 9.89 & .008 \\
\hline Lexical decision & 24.92 & $<.001$ & 46.16 & $<.001$ & 72.13 & $<.001$ \\
\hline Object decision & 9.55 & .009 & 25.70 & $<.001$ & 11.39 & .006 \\
\hline Delayed copy & 2.01 & .182 & 287.62 & $<.001$ & 0.019 & .892 \\
\hline
\end{tabular}

but little influence of familiarity. This is not especially surprising because the 24 items in this task were not chosen on the basis of a familiarity manipulation; they were merely, post hoc, divided into the higher and lower familiarity subsets of 12 each. So, with this one unsurprising exception, all of the outcomes regarding accuracy in these six tasks followed the predicted pattern of performance in SD patients. Of the 84 observations in a low-frequency irregular condition (14 patients $\times$ 6 tasks), there was not a single instance where a patient's performance was in the normal range.

\section{Errors}

Figure 2 displays, for the three language tasks involving free generation of responses, the proportions of different response types in each of the four conditions of the task. Correct responses are self-explanatory. The term LARC, coined by Patterson, Suzuki, Wydell, and Sasanuma (1995) in a study of surface dyslexia as an acronym for Legitimate Alternative Reading of Components, can be extended to tasks other than reading as Legitimate Alternative Rendering of Components. It refers to any response composed of elements that-although not all correct for this particular target stimulus - are legitimate renderings of the components of the stimulus in the sense that they are correct for those components in a different context. LARC can be considered a broader term for regularization errors. The category subsumes all errors that would, in a more classical approach, be called regularizations, but it does not assume that there is only one rule-governed way in which to respond to an atypical stimulus. Thus, for example, in reading aloud the word blood, only a response rhyming with "food" would be considered a regularization error; but a response rhyming with "good" is also treated as a LARC error. One of the advantages of the LARC classification is that it can also encompass many of the errors that patients make to regular words, as for example when a patient pronounces the written word hoot to rhyme with "foot." The third response type in Figure 2 is anything other than a correct response or an LARC error.

The striking commonality of the three panels in Figure 2 is that, as one moves across the four stimulus conditions in each task, the proportion of responses corresponding to LARC errors increases monotonically and there is a gradual tradeoff between correct responses and LARC errors. For the high-frequency regular conditions, the majority of responses were correct, and thus, of course there were scarcely any LARC errors. By contrast, once the patients were required to grapple with low-frequency irregular targets, LARC errors were as likely as correct responses for reading and verb inflection, and even substantially more likely than correct responses for spelling. Low-frequency regular and high-frequency irregular targets engendered ratios of correct/LARC responses in between the easiest and most difficult conditions. Another feature to note in Figure 2 is that non-LARC "Other" errors did not increase monotonically across the four conditions of any of the three tasks. There was a trend toward more "Other" errors for the low- than the high-frequency targets, but no hint of increased "Other" errors in the irregular relative to the regular conditions. The only additional point of interest about the "Other" errors is their somewhat higher incidence in the verb-inflection task relative to reading and even to spelling. This is because one type of "Other" error occurred in the pasttense verb task that cannot occur in reading or spelling: exact reproduction of the stimulus. In reading, subjects must translate from orthography to phonology to respond, and in spelling they must do the reverse; but in the verb task, if they do not know how to transform the verb from its stem form to its past-tense form, they can and sometimes do simply repeat the stem form that they have just heard (e.g., "Today I buy a newspaper; yesterday I buy a newspaper').

The results of the error analysis for the remaining production task, delayed copy drawing, are depicted in Figure 3. The left half of the figure shows the mean proportion of shared and distinctive features of the 


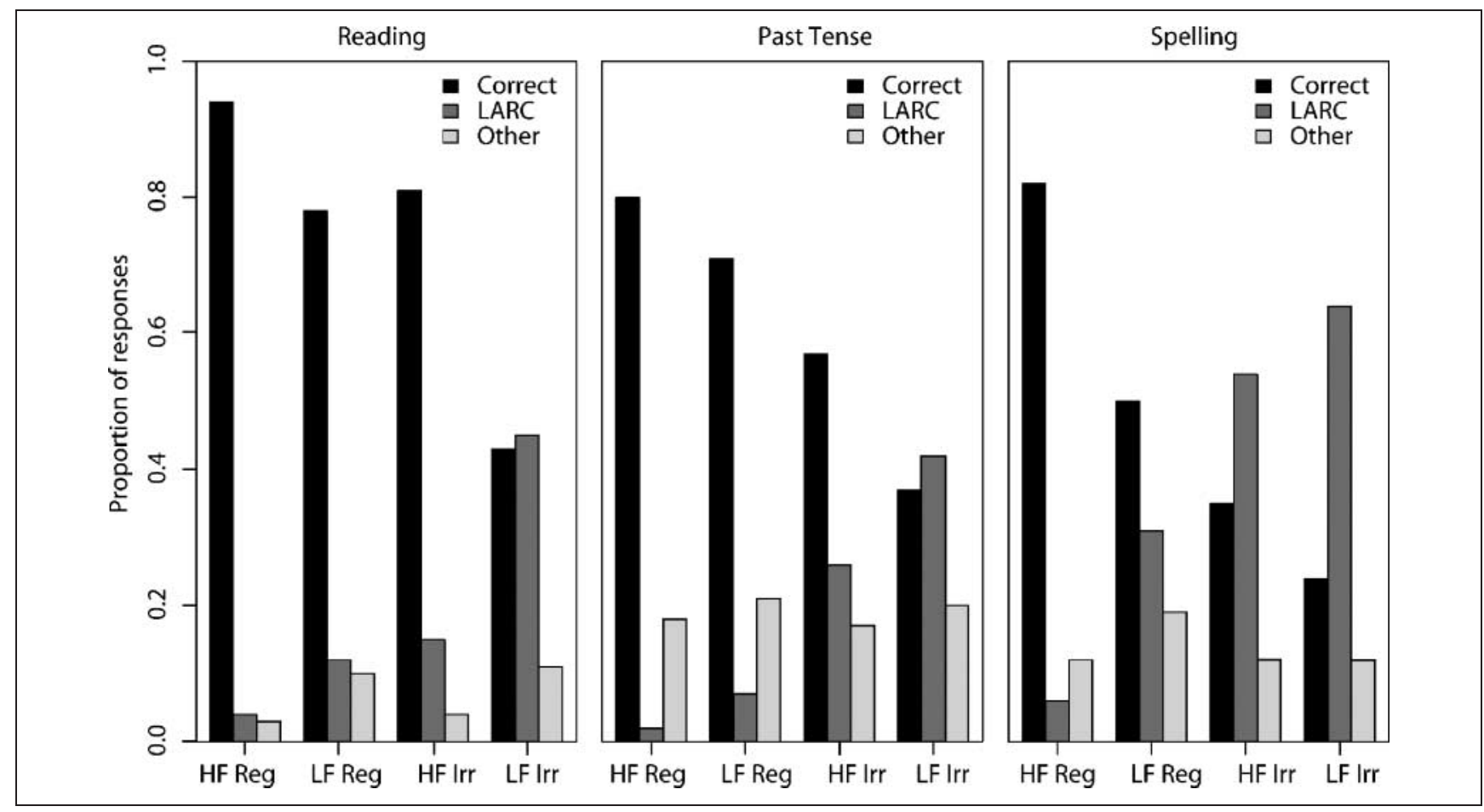

Figure 2. Proportion of responses that were correct, LARC errors (see text), or other errors in the three productive verbal tasks, plotted separately for the four task conditions.

target objects omitted in the patients' drawings, tabulated separately for the milder and more severe patient subsets. Distinctive features were much more likely to be omitted than shared features in both, with the more severe group omitting more features overall. The right side of the graph shows the mean proportion of intrusions among shared and distinctive features. Note that, by definition, there are few opportunities for shared properties to intrude: These properties tend to be common to semantically related items, and thus, can only be incorrectly added to the few items in a category that do not happen to share them. We therefore tabulated the intrusions by taking the number of shared or distinctive intrusions for each item, and dividing this by the total number of opportunities for intrusion across shared and distinctive properties for that item. The results show that intrusions are proportionately much more likely to occur for shared than for distinctive properties, in both the milder and more severe subgroups. Thus, in summary, visual properties that distinguish an item from its category neighbors are likely to be omitted, whereas visual properties that typify a given domain are likely to be added to the few domain members that do not share them.

\section{Relationship to Semantic Deficit}

Figure 4 displays (in six separate panels, one for each task) the plot of every patient's composite semantic score against his or her proportion of correct responses on the irregular target items in the relevant task. Highand low-frequency irregular items have been combined here, for two reasons. First, for some tasks (especially spelling), there were not many items per frequency condition, such that including both frequency bands provides a more stable estimate of performance. Secondly, some of the patients' scores on the lower-frequency irregular items in one or more tasks were virtually at floor; including the higher-frequency irregular items yielded a better range of scores to correlate with semantic status.

At the top of each panel, beside the task label, is the correlation between the values on the $x$ - and $y$-axes. In four of the six tasks, two different correlation values are provided: These refer to the $r$ value with/without the data points in the panel represented by symbols other than a circle. These are so-called "high-influence" data points, with a Cook's distance $\geq 0.3$ [or approximately 4/ $(n-k-1)$ where $k$ is the number of independents; see Fox, 1991] whose inclusion/exclusion alters the fit of the regression model to the remaining data points. The regression line in each panel is the least-squares fit excluding the high-influence points.

There were no high-influence data points for reading, so there is only one $r$ value here: the correlation between composite semantic score and reading irregular words was $r=.81$. There were two deviant scores for verb inflection: in both, the patient's success in inflect- 
Figure 3. Proportion of features omitted (left) or intruding (right) in patient drawings, plotted separately for less and more severe patients and for shared and distinctive features.

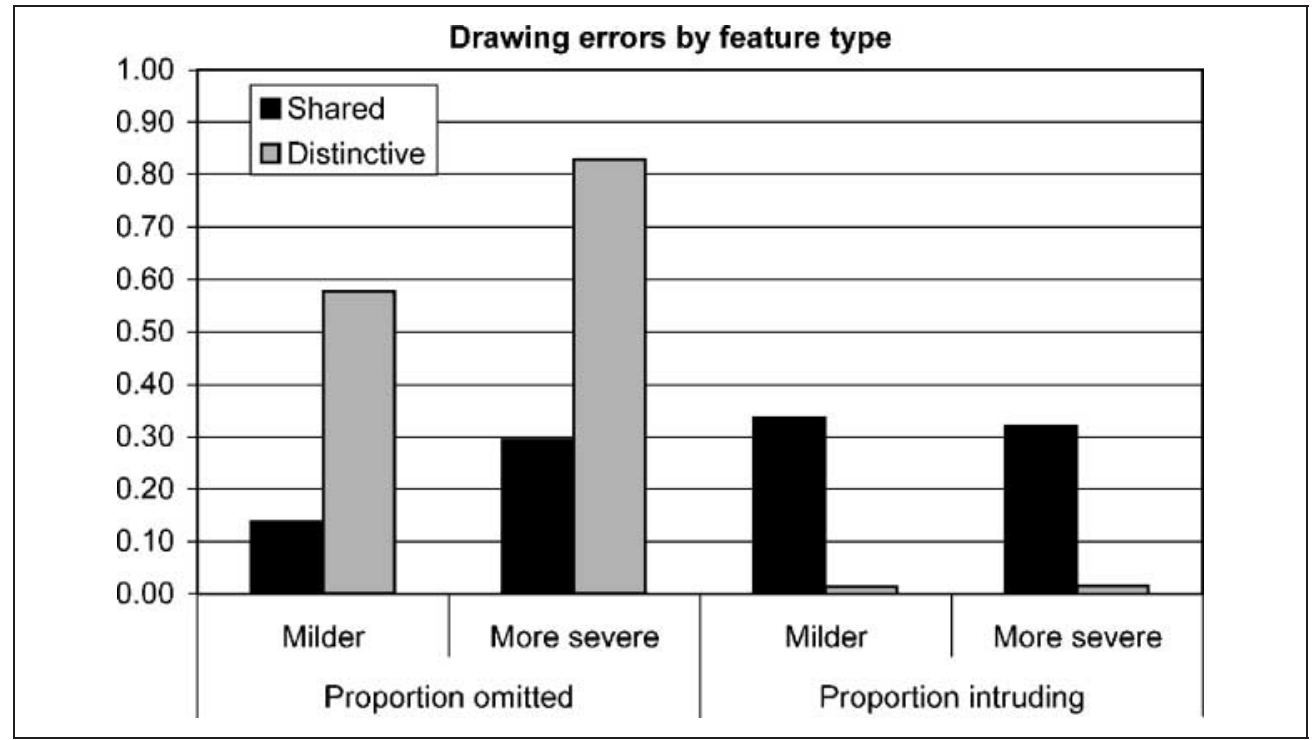

ing irregular verbs was worse than would be expected on the basis of his (LS) or her (JTh) semantic impairment. For spelling and lexical decision, there was one high-influence data point each: Patient JP, the least semantically impaired case of the 14 by the composite measure, had lower scores than predicted on these two tasks. There were no such points for object decision, the task that yielded the lowest correlation with composite semantic score. Some of the data points for object decision seem considerably above or below the regression line, but because the clustering around this line was looser in this task than the other five, the analysis did not identify any points with a Cook's distance $\geq 0.3$. For drawing, there were two deviant cases: LS was poorer than predicted for his (relatively mild) degree of semantic deficit, and WM produced better delayed copy drawings than expected for her (relatively severe) semantic decline. Summarizing across tasks, by this method of analyzing the results, there was only one instance out of a possible 84 ( 14 patients $\times 6$ tasks) where a patient's performance on irregular items (WM for delayed copy drawing) significantly exceeded the level of performance predicted by her degree of semantic deficit. In 5/84 cases, the level of success was significantly lower than predicted.

The preceding paragraph dealt with this relationship for each task across the 14 patients, but another way of addressing this issue that is germane to our hypothesis is to examine the relationship for each patient across the six tasks. This can be seen in Table 2, where the patients are listed from least to most semantically impaired by the composite measure. On the left-hand side of the table, in the six columns identified by task label, the symbols (explained in detail in the table) indicate the closeness of that patient's score for that task to the corresponding regression line in Figure 4. On the right-hand side of the table are summaries, for each patient and for the group as a whole, of the numbers of scores at varying distances from the regression lines. With the exception of JP, whose scores on the six tasks were consistently somewhat below the values predicted by his composite semantic score, every patient had a majority of scores that were either on $(\backslash)$ or very near $(+$ or -$)$ the regression line. There is not much in the way of coherent pattern to the subset of tasks on which each patient performed better or less well than predicted, with perhaps three exceptions: AN, whose temporal atrophy is left-dominant, achieved scores at or below predicted levels for the four verbal tasks but mildly better than predicted for the two visual tasks. WM-with the exception of lexical decision-showed an even stronger version of AN's pattern. She is noteworthy by virtue of the fact that her atrophy is still highly asymmetrical (and left-dominant) after $6+$ years of the disease, at a stage when the atrophy is typically extensive on both sides. Finally, BS, whose temporal atrophy is right-dominant, showed something like the reverse pattern to AN and WM, with better-than-expected scores on all of the verbal tasks and lower-than-expected values on the two visual tasks. These patterns make some sense in terms of classical hemispheric division of labor.

As a third means of understanding the relationship between a patient's degree of semantic impairment and his/her performance on the six "presemantic" tasks, we entered all of the scores shown in Figure 4-proportion correct for "irregular" items in each task-along with the composite semantic score for each patient into an exploratory factor analysis. If certain of the tests pattern reliably together, over and above their correlation with semantic impairment, the factor analysis should indicate that two or more components contribute significantly to the covariance matrix for these seven measures. The 
Figure 4. Scatterplots showing the correlation between the magnitude of semantic impairment (measured by the composite semantic score on the $x$-axis) and performance on the atypical items in each of the six tasks ( $y$-axis). High-influence data points are indicated with a triangle (for the five instances of worse-than-predicted performance) or a cross (for the one instance of better-than-expected performance). Where two correlation coefficients are shown, these reflect the strength of the relationship with and without the high-influence points included. The lines in each plot show the least-squares fit of the regression model excluding high-influence points.
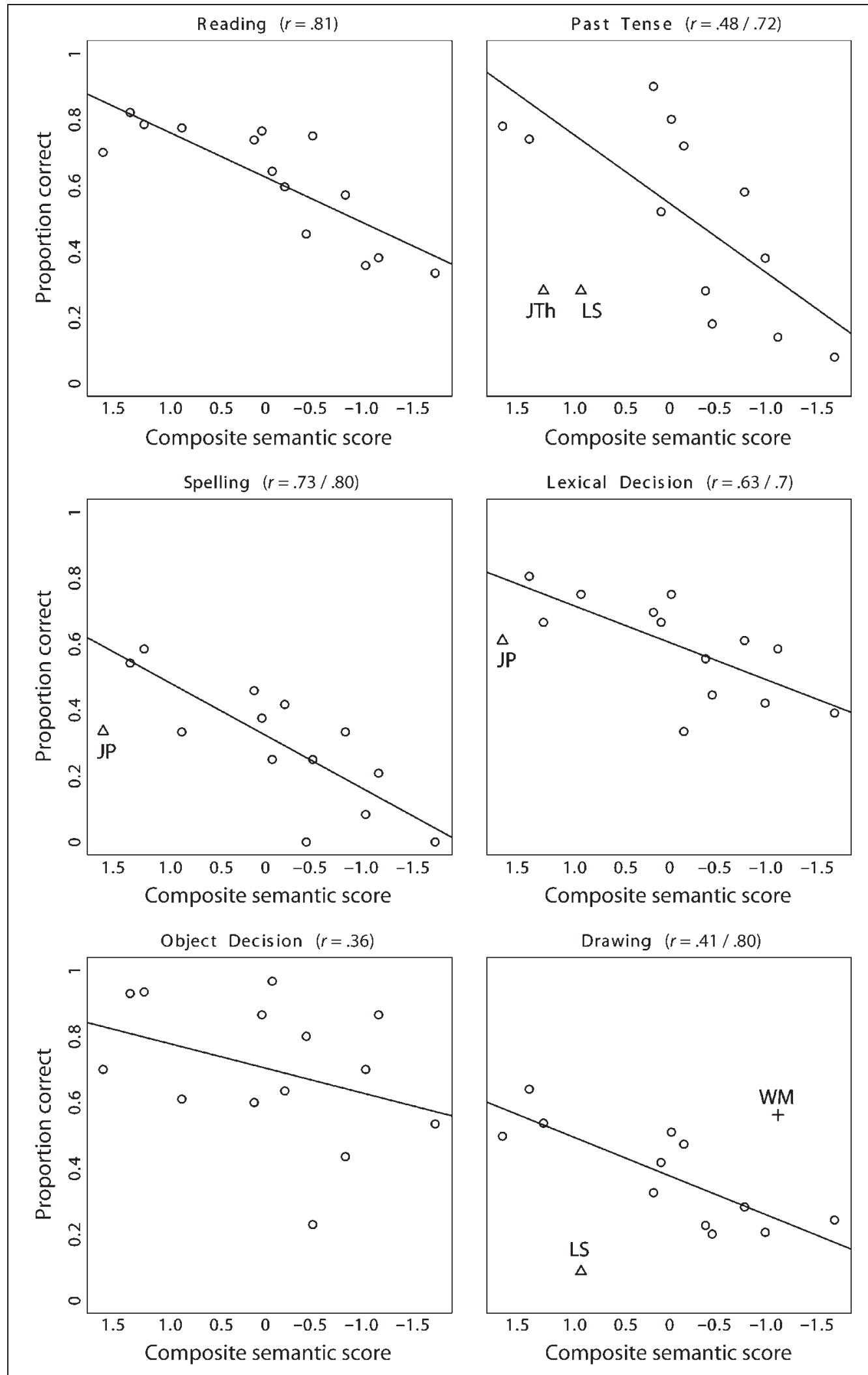

analysis was performed on the matrix derived from the raw data, as no task deviated reliably from normal. Principal components with an eigenvalue exceeding the mean were accepted in the final solution. The algorithm found a single component accounting for $87 \%$ of the variance across the seven measures. The next-largest eigenvalue was an order of magnitude smaller than the first, indicating that little nonrandom vari- 
Table 2. Proximity to Regression Line in Figure 4 for Each Patient on Every Task

\begin{tabular}{|c|c|c|c|c|c|c|c|c|c|c|c|c|c|c|}
\hline & Read & Verbs & Spell & $L D$ & $O D$ & Draw & 1 & - & + & -- & ++ & --- & +++ & \\
\hline JP & - & - & --- & --- & - & - & 0 & 4 & 0 & 0 & 0 & 2 & 0 & \\
\hline AN & 1 & - & 1 & 1 & + & + & 3 & 1 & 2 & 0 & 0 & 0 & 0 & \\
\hline JTh & $\backslash$ & --- & $\backslash$ & 1 & + & 1 & 4 & 0 & 1 & 0 & 0 & 1 & 0 & \\
\hline LS & 1 & --- & - & 1 & - & --- & 2 & 2 & 0 & 0 & 0 & 2 & 0 & \\
\hline BS & + & ++ & + & + & - & - & 0 & 2 & 3 & 0 & 1 & 0 & 0 & \\
\hline DV & + & $\backslash$ & $\backslash$ & $\backslash$ & + & $\backslash$ & 4 & 0 & 2 & 0 & 0 & 0 & 0 & \\
\hline SJ & 1 & ++ & $\backslash$ & + & ++ & + & 2 & 0 & 2 & 0 & 2 & 0 & 0 & \\
\hline EK & $\backslash$ & + & + & -- & $\backslash$ & + & 2 & 0 & 3 & 1 & 0 & 0 & 0 & \\
\hline NS & - & - & -- & $\backslash$ & + & - & 1 & 3 & 1 & 1 & 0 & 0 & 0 & \\
\hline KI & + & -- & $\backslash$ & - & -- & - & 1 & 2 & 1 & 2 & 0 & 0 & 0 & \\
\hline JTw & $\backslash$ & + & + & - & 1 & + & 2 & 1 & 3 & 0 & 0 & 0 & 0 & \\
\hline ATe & - & 1 & 1 & 1 & 1 & 1 & 5 & 1 & 0 & 0 & 0 & 0 & 0 & \\
\hline WM & 1 & - & 1 & + & ++ & +++ & 2 & 1 & 1 & 0 & 1 & 0 & 1 & \\
\hline \multirow[t]{2}{*}{$\mathrm{JG}$} & 1 & - & $\backslash$ & $\backslash$ & $\backslash$ & $\backslash$ & 5 & 1 & 0 & 0 & 0 & 0 & 0 & \\
\hline & & & & & & & 33 & 18 & 19 & 4 & 4 & 5 & 1 & 84 \\
\hline
\end{tabular}

The left-hand side of the table indicates whether each individual patient's score on each of the six experimental tasks fell: essentially right on the regression line in Figure 4 (indicated by the symbol $\backslash$ ); a little below $(-)$ or a little above $(+)$ the regression line; some, but not a significant degree, below $(--)$ or above $(++)$ the line; or a significant degree below $(---)$ or above $(+++)$ the line with a Cook's distance of $>0.3$. The right-hand side of the table summarizes for each patient and for the group as a whole, how many scores on the six tasks were located at each of these distances from the regression line relating performance to degree of semantic deficit.

ance remained to be explained in the data once the first component was considered.

\section{DISCUSSION}

The noteworthy empirical outcomes of this study can be summarized as follows. We have obtained a set of 84 observations in a low-frequency irregular condition (14 patients $\times 6$ tasks) for which we predicted impaired performance, and this prediction was correct in 84/84 cases. It is important to reiterate that these six tasks are fairly disparate in the nonsemantic processes that they require. Indeed, in a few cases, it might be difficult to argue that a pair of the tasks, such as single-word reading and delayed copy drawing, or object decision and past-tense verb inflection, would have any nonsemantic components in common. Yet all 14 patients had abnormal performance on low-frequency atypical items in both of these pairs of dissimilar tasks, as well as all the remaining tasks. It is also important to re-emphasize the selectivity of these impairments in combination with focal semantic deterioration: These patients performed normally on a number of nonsemantic tests like nonverbal reasoning, visuospatial processing, digit span, and so forth (see Table 3), and indeed, they mostly achieved good performance even on the experimental tasks when the words/objects to be recognized/produced had high familiarity and typical surface structure. The specific pattern of the impairment was the same across tasks, in two senses. First, a significant frequency-by-typicality interaction characterized success in all tasks except delayed copying, which did not have a strong itemfrequency manipulation. Second, for the four tasks involving free production, the same error type-rendering an atypical item as if it were typical-predominated in all tasks. Finally, of 84 observations of the correspondence between a patient's performance on irregular items and his or her concurrent level of semantic degradation, only $1 / 84$ represents performance that significantly alters the fit of the regression model when included and reflects better-than-expected performance given the level of semantic impairment.

The literature already contained several reports of deficits on these six tasks in patients with SD. The current investigation was designed to provide more extensive empirical observations on the association that might also adjudicate between two interpretations of the association: IAS, which proposes that the six impairments are caused by the central semantic deterioration, versus ABUD, which argues that there is no causal link between a patient's semantic status and his or her impairment in verb inflection or lexical decision or delayed copy drawing, and so forth. The account under- 
lying IAS was outlined in the Introduction; the basis for ABUD, as we understand it, is as follows.

The theoretical view in ABUD is that the modularity of the normal cognitive architecture enables all six of these tasks to be accomplished without reference to semantic information about the words or objects being processed. For reading aloud, words are translated from orthography to phonology by lexical and/or nonlexical procedures in which word meaning plays no significant part (Coltheart, Rastle, Perry, Langdon, \& Ziegler, 2001). Pasttense forms of verbs are generated from their stem forms on the basis of morphological rules (if the verbs have regular inflections) or lexical associative knowledge (if they are irregular), and again these are apparently considered to be pre- or extrasemantic procedures (Miozzo \& Gordon, 2005; Tyler et al., 2004; Miozzo, 2003; Pinker, 1999). Acceptance of a word (in lexical decision) or picture (in object decision) as real/familiar requires only contact with the appropriate node in an orthographic lexicon or structural description system, each of which precedes and indeed is a gateway to conceptual knowledge about the word or object (Coltheart, 2004; Riddoch \& Humphreys, 1987). Delayed copy drawing of familiar objects, of which there is far less discussion in the literature, presumably depends only on the structural description system plus visual short-term memory.

The empirical evidence for ABUD stems from case studies of patients with semantic impairments who perform normally on one or more of the various "extrasemantic" tasks considered here. We will consider exceptional case studies and their implications for ABUD and IAS in further detail below. Here we simply note that, for proponents of ABUD, such cases conclusively refute the hypothesis that extrasemantic deficits in SD are caused by an underlying semantic impairment. On this view, if good performance is observed on such a task when semantic knowledge is degraded, even for $N=1$ case, there can be no causal link, no matter how large the $N$ of cases demonstrating an association (see, for example, Coltheart, 2004). The association is treated as a coincidence arising from concurrent damage to functionally unrelated brain regions.

Our challenges to ABUD on the basis of the data presented here are as follows: (i) At this stage of knowledge in our field, we have only tentative hypotheses about the neuroanatomical regions required for performance of these six tasks, and indeed, about the regions involved in the representation of conceptual knowledge. Nevertheless, we ask how likely it is that the spread of atrophy outwards from the temporal pole, where the pathological process in SD typically begins, would happen to affect the disparate sites required for all six tasks in every patient. (ii) We further ask how likely it is that the degree of impairment in every task should be modulated by the degree of semantic degradation. Suppose that the orthographic lexicon or visual word-form system, upon which written-word lexical decision may be based (Coltheart, 2004), is located in the left occipito-temporal region (McCandliss, Cohen, \& Dehaene, 2003; Dehaene, LeClec'H, Poline, Le Bihan, \& Cohen, 2002). It is perhaps plausible that atrophy starting in the anterior left temporal lobe might spread caudally to affect this posterior temporal site, such that an emerging deficit in lexical decision would coincidentally correlate with an increasing semantic deficit. But how would one also explain that the same patient's degree of semantic deficit concurrently predicts the degree of deficit in delayed copy drawing? The nonsemantic components of this task are probably a structural description system thought to be localized around the right posterior temporal/inferior parietal region (Gerlach, Law, Gade, \& Paulson, 1999; Warrington \& Taylor, 1973) and/or visuospatial short-term memory which might depend on lateral frontal structures (Bor, Duncan, Wiseman, \& Owen, 2003). (iii) Even if the answers to (i) and (ii) were "not impossible," we ask how likely it is that the pattern of impairment across all tasks would be so similar, with accuracy characterized by a frequency-by-typicality interaction and errors almost always more typical of the domain than the target. If deficits in these "nonsemantic" functions were only accidentally associated with, and truly unrelated to, the semantic disintegration, then why, for example, don't some patients have phonological rather than surface dyslexia? Or, if they make mistakes in 2AFC lexical decision, why don't a few of them reveal the pattern demonstrated in Rogers, Lambon Ralph, Hodges, and Patterson (2004) for stroke patients with Broca's aphasia, who were impaired in the task and worse for lowfrequency targets, but were completely unaffected by the orthographic typicality manipulation?

Our own theoretical account, which is summarized in the Introduction and more extensively described in Rogers, Lambon Ralph, Garrard, et al. (2004), yields the following answer to each of the three challenges just outlined. (i) All six tasks are impaired by atrophy confined to the anterior temporal lobe because, in addition to all of the disparate regions involved in the nonsemantic components of the tasks, all require interaction with the high-level semantic representations based on this brain region. (ii) In addition to the frequency and typicality of the input, the best predictor of a patient's success in all tasks will be his or her degree of semantic degradation because none of the tasks is truly non- or pre-semantic. (iii) Damage to this single semantic system will have the same pattern of impact on all of these tasks - a frequency-by-typicality interactionbecause lower-frequency atypical items receive the least support from knowledge of modality-specific surface structure, and thus, depend to a greater extent on support from semantics.

We acknowledge that there is evidence from SD, for at least a few of the six tasks, to support the view that 
these processes do not need input from/interaction with semantic knowledge in order to deliver correct responses, even for atypical lower-frequency stimuli. That is, there have been a few single-case reports of SD patients who were not impaired at the task in question. In some cases, it seems likely that the apparently spared performance derives from test materials that were not structured in the manner required to reveal the deficit. If the nonreal objects in object decision are less typical than the real objects, for example, the patients will perform well, as illustrated in Figure 1 (and explored at greater length in Rogers, Lambon Ralph, Hodges, \& Patterson, 2003, 2004). In other cases, absence of a recorded deficit may simply derive from lack of power to detect it. Tyler et al. (2004) recently described four SD patients tested on a past-tense elicitation task similar to the one described here, and reported perfect success for two of the patients on a set of 22 irregular verbs (13 higher frequency and 9 lower). As it happens, three of the four patients in Tyler et al., although only one of the two with perfect scores on irregular verbs, also participated in our study at around the same time. Tyler (personal communication) kindly provided us with her materials: There are nine specific past-tense irregular verbs (such as came, chose, drank) that appeared in both their set of 22 and our set of 50 irregular verbs. On these nine overlapping items, there is no more than a single item of discrepancy in any of the three patients' scores. In other words, although the results of the two studies seem to conflict, they do not; and the most likely reason for the apparent discrepancy is simply that our study employed more than twice as many items in the general irregular set and nearly three times as many in the critical set of low-frequency irregular items, with a consequent greater sensitivity to detecting a deficit.

Nevertheless, there are a few reports of unimpaired performance on one of our six tasks, in single cases of $\mathrm{SD}$, that cannot be explained by insensitivity of the test. To our knowledge, three such cases exist for reading aloud (Blazely, Coltheart, \& Casey, 2005; Cipolotti \& Warrington, 1995; Schwartz, Saffran, \& Marin, 1980), ${ }^{1}$ although WLP, the patient reported in Schwartz et al., subsequently developed the pattern of surface dyslexic reading predicted and reported in the current manuscript. The literature also reports one SD case with unimpaired written-word lexical decision for materials that have been matched for orthographic typicality (Blazely et al., 2005). We are not aware of any convincing demonstrations of preserved ability for the other four tasks in SD, but they may be just around the corner. The question, of course, is whether these very occasional dissociations constitute critical evidence against the IAS account of the association. By traditional neuropsychological argument, the answer to this question is yes. The claim is that spurious associations can arise when otherwise unrelated cognitive functions happen to depend upon neural structures that are jointly compromised in pathology-most likely because they are neuroanatomical neighbors. Dissociations, by contrast, are considered straightforwardly to disprove causality.

It will be obvious by now that, although this selective attention to dissociations is a widely held view, we do not share it. The processes that support word reading, spelling, drawing, and so on, are complex and subject to many sources of variation, so that pathological conditions such as SD will produce a distribution of outcomes in these abilities. The current data document a strong population-level association between the extent of semantic impairment and degree of deficit on atypical items in each of the tasks we have studied, but in no case is this relationship perfect: There is always variation around the regression line (see Lambon Ralph, 2004; Lambon Ralph, Moriarty, \& Sage, 2002 for further discussion of this point). If this variation is distributed normally, then a small number of cases will fall in the upper tail of the distribution, performing better than expected given their level of semantic impairment; in the rare case, "better than expected" may even be within the normal range. Although it is certainly important to work towards an understanding of why a few individuals fall in the extremes of the distribution, we nevertheless argue that selective emphasis on these exceptional cases is misguided because it fails to explain the majority of the evidence. As indicated above, the undifferentiated response of "accidental association due to anatomical proximity" is not an explanation for the data presented here from tasks so varied that they must rely on far-flung brain regions. A single factor-presumably degree of semantic degradation-accounts for an impressively high $87 \%$ of the variance in performance on both our semantic measures and our six cognitively and anatomically disparate "presemantic" tasks. Our theory predicted not only this observed association, but also the precise pattern of the deficit obtained in all six tasks.

In conclusion, the six deficits or "characters" searching for an explanation in this play have found one: They are grouped into one story by a single, semantic author.

\section{METHODS}

\section{Participants}

The SD cohort consisted of 14 patients who were initially seen by a senior neurologist/physician and a clinical neuropsychologist in UK hospital clinics in either Cambridge or Bath. In addition to a clinical and neurological assessment, each patient was given a number of standard psychiatric rating scales to exclude major psychiatric disorders such as depression and schizophrenia, as well as structural brain imaging and the usual battery of screening blood tests to exclude treatable causes of dementia. All patients fulfilled the international consensus and local criteria for SD (Neary et al., 1998; Hodges, Patterson, Oxbury, \& Funnell, 1992): impaired receptive 
and expressive content-word vocabulary and impoverished semantic knowledge, with relative preservation of nonverbal reasoning, visuospatial abilities, phonology, syntax and day-to-day memory, plus structural brain imaging revealing focal atrophy in anterior, inferior regions of the temporal lobe. Each of these cases has already been included in another publication on SD (for example, all were among the 22 cases in Rogers, Lambon Ralph, Hodges, et al., 2004), so we have not provided detailed descriptions. Demographic characteristics and some basic background neuropsychological data are given in Table 3. In the table, the patients are listed in the order from least (JP) to most (JG) semantically impaired as measured by a composite semantic score which appears in the last column of Table 3; its calculation is described below.

In Table 3, scores on the Mini-Mental State Examination are given as an indication of general cognitive status. Scores on both copying the complex Rey Figure and the Cube Analysis subtest from the Visual Object and Space Perception battery (Warrington \& James, 1991) are included to indicate that none of the patients had a visuospatial deficit. Cube analysis requires the participants to count the cubes in a 2-D picture of a 3-D array of cubes; some of the cubes necessary to support the pictured structure are not visible, and the test therefore requires visuospatial reasoning as well as perception. Forward digit span demonstrates that none of the patients had a significant impairment of auditory-verbal working memory, with a number of patients-even those with more severe semantic deficits-reliably able to reproduce a sequence of eight digits. All patients but one were tested on Raven's Coloured Progressive Matrices as an assessment of nonverbal, nonsemantic reasoning ability. Two cases had poor scores (BS $\sim 50$ th percentile for his age, and $\mathrm{KI} \sim 25$ th); but all of the other patients performed very well on this test, and BS and KI were not the most severely impaired on semantic measures and had normal performance on other nonsemantic tests such as Rey Copy, Cube Analysis, and Digit Span.

The next three tests, on which normal individuals score essentially perfectly, are ones always used in our research as assessments of semantic memory: naming of line drawings of familiar objects for the 64 items in the Cambridge semantic battery; spoken word-to-picture matching (WPM) on the same 64 items, where the response arrays consist of the target picture along with nine other objects from the same semantic category; and the all-picture version of the Pyramids and Palm Trees (PPT pics) test of associative semantic knowledge (Howard \& Patterson, 1992). For 11 patients, all three semantic measures were taken within 6 months of testing on the six tasks of interest here. For the remaining three patients, testing on the six central tasks occurred in between two regular (approximately yearly) longitudinal assessments on the semantic battery; for these patients, a

Table 3. Demographic Characteristics and Basic Background Neuropsychology for the 14 Patients Participating in this Study

\begin{tabular}{|c|c|c|c|c|c|c|c|c|c|c|c|}
\hline & Sex & Age & MMSE & Rey Copy & $\begin{array}{c}\text { Cube } \\
\text { Analysis }\end{array}$ & Digit Span & $\begin{array}{c}\text { Raven's } \\
C P M\end{array}$ & Naming & WPM & PPT pics & $\begin{array}{c}\text { Composite } \\
\text { Semantic }\end{array}$ \\
\hline Max score & $\mathrm{N} / \mathrm{A}$ & N/A & 30 & 36 & 10 & N/A & 36 & 64 & 64 & 52 & N/A \\
\hline \multicolumn{12}{|l|}{ Patient } \\
\hline JP & $\mathrm{M}$ & 66 & 26 & 36 & 10 & 5 & 36 & 49 & 63 & 47 & 1.67 \\
\hline $\mathrm{AN}$ & M & 65 & 27 & 36 & 9 & 8 & 35 & 41 & 62 & 48 & 1.4 \\
\hline JTh & $\mathrm{F}$ & 55 & 25 & 31 & 10 & 6 & NT & 43 & 55 & 49 & 1.26 \\
\hline LS & $\mathrm{M}$ & 61 & 24 & 29 & 9 & 7 & 31 & 34 & 60 & 29 & 0.88 \\
\hline BS & $\mathrm{M}$ & 68 & 25 & 33 & 10 & 8 & 25 & 29 & 40 & 33 & 0.16 \\
\hline DV & $\mathrm{M}$ & 65 & 23 & 36 & 10 & 8 & 31 & 15 & 49 & 41 & 0.08 \\
\hline SJ & $\mathrm{F}$ & 60 & 19 & 34 & 10 & 5 & 34 & 11 & 51 & 45 & -0.02 \\
\hline EK & $\mathrm{F}$ & 60 & 27 & 35 & 10 & 7 & 33 & 17 & 43 & 33 & -0.15 \\
\hline NS & $\mathrm{F}$ & 69 & 25 & 36 & 10 & 5 & 36 & 8 & 42 & 39 & -0.37 \\
\hline KI & $\mathrm{M}$ & 65 & 23 & 34 & 10 & 8 & 21 & 15 & 36 & 31 & -0.43 \\
\hline $\mathrm{JTw}$ & $\mathrm{M}$ & 66 & 25 & 31 & 10 & 8 & 36 & 5 & 34 & 35 & -0.76 \\
\hline ATe & $\mathrm{M}$ & 62 & 20 & 36 & 10 & 8 & 34 & 3 & 29 & 31 & -0.97 \\
\hline WM & F & 55 & 21 & 34 & 10 & 5 & 35 & 6 & 21 & 38 & -1.09 \\
\hline JG & $\mathrm{F}$ & 71 & 19 & 34 & 9 & 6 & 34 & 2 & 8 & 35 & -1.66 \\
\hline
\end{tabular}


more time-appropriate estimated score on each of the three semantic measures was created by averaging the scores from the testing rounds immediately preceding and following the time of the experimental testing.

Scores on the three semantic tests were used to create an estimate of each patient's degree of deterioration in conceptual knowledge. Of these tests, naming is most sensitive to mild semantic impairment, whereas WPM and PPT pics are more sensitive to moderate and severe impairment at a point when object naming is often at floor. To derive a composite score indexing overall degree of semantic impairment, we conducted a factor analysis on the three semantic measures. Given that none of the measures deviated significantly from normal $(p>.05)$, the raw scores were entered directly into the analysis. Principal components were extracted from the covariance matrix and vectors with eigenvalues exceeding the mean were accepted in the final solution. The analysis yielded a single factor that accounted for $84 \%$ of the variance in the three tests, and the eigenvalue of the second component was an order of magnitude smaller than the first. In other words, a common underlying factor accounts for most of the variance and covariance among the three semantic tests, with additional factors providing little explanatory power. Scores on the single composite semantic factor were then calculated for each of the 14 patients (see the last column of Table 3). The composite score has a mean of zero and unit variance, so that positive values indicate milder-than-average impairments, whereas negative values indicate impairments that are more severe than average.

We had results from age- and education-matched healthy individuals for each of the six experimental tasks (with identical materials) from previous research, and the claim about the importance of data on all tasks from the same participants does not seem to apply to normal individuals; therefore, no new control data were collected for this study. The control data to which the patients' performance was compared come from the following published studies: for both reading aloud and spelling to dictation, with $n=24$ normal participants each, Graham et al. (2000); for verb inflection with $n=10$, Patterson et al. (2001); for both lexical decision $(n=11)$ and object decision $(n=10)$, Rogers, Lambon Ralph, Hodges, et al. (2004); and for delayed copy drawing $(n=4)$, Bozeat et al. (2003).

\section{Stimulus Materials and Procedures}

The reading test consisted of 168 single-syllable words, from three to six letters in length, with 42 items in each of the four conditions formed by crossing word frequency (high vs. low) and regularity of spelling-sound correspondences (regular vs. irregular). This is the "surface" list (Patterson \& Hodges, 1992) but with the medium-frequency band of words removed to make the test a little shorter and quicker to administer. The words were typed onto cards and a random ordering of the cards was presented to each participant with the instruction to read each word aloud.

The spelling test consisted of 36 single-syllable words (a subset of the surface list: Patterson \& Hodges, 1992). There were six words in each of the three conditions formed by crossing frequency (high, medium, low) with regularity (regular vs. irregular). For purposes of analysis, because all of the other tests in this study had only high- and low-frequency conditions, the medium- and low-frequency words in each of the regularity conditions were combined into a low-frequency set, thus giving 6 high-frequency words and 12 lower-frequency items in each regularity condition. For test administration, the experimenter dictated each word (with the list in random order) and first asked the patient to repeat the item to ensure that it had been heard correctly.

The verb inflection test consisted of the 100 verbs from Patterson et al. (2001), with 25 items in each of the four conditions formed by crossing word frequency (high vs. low) and regularity of past-tense structure (regular vs. irregular). For administration of the test, the participant first simultaneously heard and saw a short sentence in which the target verb was used in its stem/present-tense form, for example, "Today I eat lunch." He or she was then immediately presented, again simultaneously in both modalities, with another sentence containing a gap where the verb should be, and was instructed to supply the spoken form of the same verb as in the first sentence, but now in the form indicating a past event (e.g., "Yesterday I lunch"). A practice set of materials (using none of the 100 experimental targets) was administered before the proper test began to ensure that the patient understood the task.

The lexical decision test was identical to the one described by Rogers, Lambon Ralph, Hodges, et al. (2004); all of the items are listed in an appendix in that article. It is a $2 \mathrm{AFC}$ test consisting of 72 pairs, each containing one real word and one nonword. In each pair, the nonword is a possible pseudohomophone of the word. The frequency manipulation in this test refers, perhaps obviously, only to the word member of each pair. The regularity manipulation refers not to the word or the nonword but to the relationship between them. In condition $\mathrm{W}>\mathrm{NW}$ (example node/gnode), the word is more orthographically typical than the nonword, operationally defined in terms of mean bigram and trigram frequencies. In condition $\mathrm{NW}>\mathrm{W}$ (example gnome/nome), the nonword is more orthographically typical than the word, again in terms of bigram/trigram frequencies. For administration of the test, the 72 pairs were each printed on a separate sheet of paper, with the real word to the left of the nonword in half of the trials and to its right in the other half, counterbalanced for condition. Participants were instructed to "point to the real word" on each sheet. 
The object decision test was also identical to the one described (again with the items listed in an appendix) by Rogers, Lambon Ralph, Hodges, et al. (2004). It is a 2AFC test consisting of 60 pairs, each containing one line drawing of a real object and one drawing of a nonreal version of the same object. The manipulation of frequency here was based on familiarity ratings of the objects (see Rogers, Lambon Ralph, Hodges, et al., 2004, p. 343). Paralleling the lexical decision test, the manipulation of regularity was in terms of the relative typicality of the real versus the nonreal version of each object. In condition R > NR (e.g., a monkey with normal monkey-sized ears vs. a monkey with large elephant-sized ears), the real version is more typical of its domain than the nonreal version, because the majority of animals have small-ish ears and elephants are atypical in this regard. In condition NR > R (e.g., an elephant with monkey-sized ears vs. an elephant with elephant ears), it is the real object that has the atypical feature. For administration of the test, the 60 picture pairs were each printed on a separate sheet of paper, with the real object to the left of the nonobject in half of the trials and to its right in the other half, counterbalanced for condition. Participants were instructed to "point to the real thing" on each sheet.

In the delayed copy test, each of 24 line drawings (a subset of the 64 drawings used in the Cambridge semantic battery) was presented individually for about $5 \mathrm{sec}$ for the participant to study; it was then taken away and the subject was asked to count from 1 to 15 (patients with SD have no trouble counting, and they, like controls, take on average about $10 \mathrm{sec}$ to do this). At the end of the delay, the patient was asked to "draw the picture that you were just looking at." Note that in this test there is never any reference to the name of the object, just "look at this; count; draw what you saw." The procedure for scoring was the one developed by Bozeat et al. (2003), in which the patients were evaluated for each drawing on their inclusion of the target features that were produced by all control subjects. For example, the picture of a duck shows two legs, and all controls reproduced a duck with two legs. If a patient drew two legs, this was counted as correct; if he drew a legless duck, this was considered a feature omission; if he drew a duck with four legs, this was considered a feature intrusion error. Target features for each drawing were further classified as shared or distinctive, based on whether they tended to be present in other semantically related items. Shared properties were common to more than half of the items in the same intermediate or superordinate category; for instance, both eyes and wings were classified as shared for the duck. Any remaining properties were classified as distinctive-for instance, the hump on the camel (see Bozeat et al., 2003 for further details). For each drawing we then calculated the proportion of features omitted or intruding, tabulating these separately for the shared and distinctive features in each drawing.
Reprint requests should be sent to Karalyn Patterson, MRC Cognition \& Brain Sciences Unit, 15 Chaucer Road, Cambridge CB2 2EF, UK, or via e-mail: karalyn.patterson@mrc-cbu. cam.ac.uk. Or to Timothy T. Rogers, Department of Psychology, University of Wisconsin, Madison, WI 53706, or via e-mail: ttrogers@wisc.edu.

\section{Note}

1. Note that we are not including here a few reported cases of preserved reading of irregular words combined with "semantic impairment" in Alzheimer's disease or transcortical aphasia from stroke (e.g., Gerhand, 2001; Lambon Ralph, Ellis, \& Franklin, 1995). This is because we share the view of Silveri and Colosimo (1995) that semantic deficits measured (as they usually are) by multiple-item forced-choice tests like wordpicture matching can significantly overestimate the semantic impairment in cases with these etiologies. A similar analysis may apply to case VP, studied by Miozzo and Gordon (2005), who was unimpaired both in reading irregular words and inflecting irregular verbs. Her semantic deficit—significant but mild relative to most SD patients-was caused by cerebral hemorrhage and was accompanied by a substantial impairment to working memory that might well interfere with the oddman-out tests used to assess her semantic processing.

\section{REFERENCES}

Blazely, A., Coltheart, M., \& Casey, B. J. (2005). Semantic dementia with and without surface dyslexia. Cognitive Neuropsychology, 22, 695-717.

Bor, D., Duncan, J., Wiseman, R. J., \& Owen, A. M. (2003). Encoding strategies dissociate prefrontal activity from working memory demand. Neuron, 37, 361-367.

Bozeat, S., Lambon Ralph, M. A., Graham, K. S., Patterson, K., Wilkin, H., Rowland, J., Rogers, T. T., \& Hodges, J. R. (2003). A duck with four legs: Investigating the structure of conceptual knowledge using picture drawing in semantic dementia. Cognitive Neuropsychology, 20, $27-47$.

Breedin, S. D., Saffran, E. M., \& Coslett, H. B. (1994). Reversal of the concreteness effect in a patient with semantic dementia. Cognitive Neuropsychology, 11, 617-660.

Bub, D., Cancelliere, A., \& Kertesz, A. (1985). Whole-word and analytic translation of spelling to sound in a nonsemantic reader. In K. Patterson, J. C. Marshall, \& M. Coltheart (Eds.), Surface dyslexia (pp.15-34). Hove, UK: Erlbaum.

Cipolotti, L., \& Warrington, E. K. (1995). Semantic memory and reading abilities: A case report. Journal of the International Neuropsychological Society, 1, 104-110.

Coltheart, M. (2004). Are there lexicons? Quarterly Journal of Experimental Psychology, 57A, 1153-1171.

Coltheart, M., Rastle, K., Perry, C., Langdon, R., \& Ziegler, J. (2001). DRC: A dual route cascaded model of visual word recognition and reading aloud. Psychological Review, 108, 204-256.

Cortese, M. J., Balota, D. A., Sergent-Marshall, S. D., Buckner, R. L., \& Gold, B. T. (in press). Consistency and regularity in past tense verb generation in healthy aging, Alzheimer's disease, and semantic dementia. Cognitive Neuropsychology.

Dehaene, S., Le Clec'H, G., Poline, J. B., Le Bihan, D., \& Cohen, L. (2002). The visual word form area: A prelexical representation of visual words in the fusiform gyrus. NeuroReport, 13, 321-325. 
Diesfeldt, H. F. A. (1992). Impaired and preserved semantic memory functions in dementia. In L. Backman (Ed.), Memory functioning in dementia (pp. 227-263). Amsterdam: Elsevier.

Fox, J. (1991). Regression diagnostics. Quantitative Applications in the Social Sciences Series No. 79. Thousand Oaks, CA: Sage Publications.

Funnell, E. (1996). Response biases in oral reading: An account of the co-occurrence of surface dyslexia and semantic dementia. Quarterly Journal of Experimental Psychology, 49A, 417-446.

Fushimi, T., Komori, K., Ikeda, M., Patterson, K., Ijuin, M., \& Tanabe, H. (2003). Surface dyslexia in a Japanese patient with semantic dementia: Evidence for similarity-based orthography-to-phonology translation. Neuropsychologia, 41, 1644-1658.

Gerhand, S. (2001). Routes to reading: A report of a non-semantic reader with equivalent performance on regular and exception words. Neuropsychologia, 39, 1473-1484.

Gerlach, C., Law, I., Gade, A., \& Paulson, O. B. (1999). Perceptual differentiation and category effects in normal object recognition: A PET study. Brain, 122, 2159-2170.

Graham, N. L., Patterson, K., \& Hodges, J. R. (2000). The impact of semantic memory impairment on spelling: Evidence from semantic dementia. Neuropsychologia, 38, 143-163.

Hodges, J. R., Patterson, K., Oxbury, S., \& Funnell, E. (1992). Semantic dementia: Progressive fluent aphasia with temporal lobe atrophy. Brain, 115, 1783-1806.

Hovius, M., Kellenbach, M. L., Graham, K. S., Hodges, J. R., \& Patterson, K. (2003). What does the object decision task measure? Reflections on the basis of evidence from semantic sementia. Neuropsychology, 17, 100-107.

Howard, D., \& Patterson, K. (1992). Pyramids and palm trees: A test of semantic access from pictures and words. Bury St Edmunds, Suffolk: Thames Valley Test Company.

Joanisse, M. F., \& Seidenberg, M. S. (1999). Impairments in verb morphology after brain injury: A connectionist model. Proceedings of the National Academy of Sciences, U.S.A., 96, 7592-7597.

Lambon Ralph, M. A. (2004). Reconnecting cognitive neuropsychology: Commentary on Harley's "Does cognitive neuropsychology have a future?" Cognitive Neuropsychology, 21, 31-35.

Lambon Ralph, M. A., Ellis, A. W., \& Franklin, S. (1995). Semantic loss without surface dyslexia. Neurocase, 1 , 363-369.

Lambon Ralph, M. A., \& Howard, D. (2000). Gogi aphasia or semantic dementia? Simulating and assessing poor verbal comprehension in a case of progressive fluent aphasia. Cognitive Neuropsychology, 17, 437-465.

Lambon Ralph, M. A., Moriarty, L., \& Sage, K. (2002). Anomia is simply a reflection of semantic and phonological impairments: Evidence from a case-series study. Aphasiology, 16, 56-82.

McCandliss, B. D., Cohen, L., \& Dehaene, S. (2003). The visual word form area: Expertise for reading in the fusiform gyrus. Trends in Cognitive Sciences, 7, 293-299.

Miozzo, M. (2003). On the processing of regular and irregular forms of verbs and nouns: Evidence from neuropsychology. Cognition, 87, 101-127.

Miozzo, M., \& Gordon, P. (2005). Facts, events, and inflection: When language and memory dissociate. Journal of Cognitive Neuroscience, 17, 1074-1086.

Moss, H. E., Tyler, L. K., Hodges, J. R., \& Patterson, K. E. (1995). Exploring the loss of semantic memory in semantic dementia: Evidence from a primed monitoring study. Neuropsychology, 9, 16-26.

Neary, D., Snowden, J. S., Gustafson, L., Passant, U., Stuss, D., Black, S., Freedman, M., Kertesz, A., Robert, P. H., Albert, M., Boone, K., Miller, B. L., Cummings, J., \& Benson, D. F. (1998). Frontotemporal lobar degeneration: A consensus on clinical diagnostic criteria. Neurology, 51, 1546-1554.

Parkin, A. J. (1993). Progressive aphasia without dementia-A clinical and cognitive neuropsychological analysis. Brain and Language, 44, 201-220.

Patterson, K., \& Hodges, J. R. (1992). Deterioration of word meaning: Implications for reading. Neuropsychologia, 30, 1025-1040.

Patterson, K., Lambon Ralph, M. A., Hodges, J. R., \& McClelland, J. L. (2001). Deficits in irregular past-tense verb morphology associated with degraded semantic knowledge. Neuropsychologia, 39, 709-724.

Patterson, K., Suzuki, T., Wydell, T., \& Sasanuma, S. (1995). Progressive aphasia and surface alexia in Japanese. Neurocase, 1, 155-166.

Pinker, S. (1999). Words and rules. London: Weidenfeld \& Nicholson.

Plaut, D. C., McClelland, J. L., Seidenberg, M. S., \& Patterson, K. (1996). Understanding normal and impaired word reading: Computational principles in quasi-regular domains. Psychological Review, 103, 56-115.

Riddoch, M. J., \& Humphreys, G. W. (1987). Visual object processing in optic aphasia: A case of semantic access agnosia. Cognitive Neuropsychology, 4, 131-185.

Rogers, T. T., Lambon Ralph, M. A., Garrard, P., Bozeat, S., McClelland, J. L., Hodges, J. R., \& Patterson, K. (2004). The structure and deterioration of semantic memory: A neuropsychological and computational investigation. Psychological Review, 111, 205-235.

Rogers, T. T., Lambon Ralph, M. A., Hodges, J. R., \& Patterson, K. (2003). Object recognition under semantic impairment: The effects of conceptual regularities on perceptual decisions. Language and Cognitive Processes, 18, 625-662.

Rogers, T. T., Lambon Ralph, M. A., Hodges, J. R., \& Patterson, K. (2004). Natural selection: The impact of semantic impairment on lexical and object decision. Cognitive Neuropschology, 21, 331-352.

Rogers, T. T., \& McClelland, J. L. (2004). Semantic cognition: A parallel distributed processing approach. Cambridge: MIT Press.

Schwartz, M. F., Saffran, E. M., \& Marin, O. S. M. (1980). Fractionating the reading process in dementia: Evidence for word-specific print-to-sound associations. In M. Coltheart, K. E. Patterson, \& J. C. Marshall (Eds.), Deep dyslexia (pp. 259-269). London: Routledge \& Kegan Paul.

Silveri, M. C., \& Colosimo, C. (1995). Hypothesis on the nature of comprehension deficit in a patient with transcortical mixed aphasia with preserved naming. Brain and Language, 49, 1-26.

Tyler, L. K., Stamatakis, E. A., Jones, R., Bright, P., Acres, K., \& Marslen-Wilson, W. D. (2004). Deficits for semantics and the irregular past tense: A causal relationship? Journal of Cognitive Neuroscience, 16, 1-14.

Warrington, E. K., \& James, M. (1991). The visual object and space perception battery. Bury St Edmunds: Thames Valley Test Company.

Warrington, E. K., \& Taylor, A. M. (1973). The contribution of the right parietal lobe to object recognition. Cortex, 9, 152-164. 